\title{
Enhanced Power Control Algorithm in Cognitive Radio for Multimedia Communication
}

\author{
C. T. Manimegalai*, K. Kalimuthu, Sabitha Gauni and R. Kumar \\ Department of Telecommunication Engineering and ECE, SRM University, Chennai - 603203, Tamil Nadu, India; \\ manimegalai.c@ktr.srmuniv.ac.in, kalimuthu.k@ktr.srmuniv.ac.in, \\ sabitha.g@ktr.srmuniv.ac.in, kumar.r@ktr.srmuniv.ac.in
}

\begin{abstract}
Background/Objectives: In underlay cognitive radio network, the power control systems play a major role where the secondary users should not affect the primary users transmission. The interference constraints are not attained by all users as the Signal to Interference Noise Ratio (SINR) of the target requirement is not satisfied in the distributed algorithm for power control. Hence, an algorithm is proposed to reduce the power and to regulate the interference constraints of the primary and secondary users. The SINR also has to be maintained. In the proposed method, the distributed statistical algorithm is used when the interference constraints is not available when all transmitters are in active mode to obtain an efficient solution. Methods: In order to minimize the level of SINR for each link between primary and secondary user, a distributed algorithm for power control is proposed. The traditional power control algorithms are used to maintain the secondary users transmission within specific power levels. The main drawback of the traditional algorithms is that the constraints are not met when all the transmitters are active. Result: A Power minimizing algorithm along with the distributed statistical elimination algorithm, to suppress the transmission of selected number of links when all the nodes are active is proposed. This satisfies the SINR requirement of the secondary links. Application: The proposed elimination algorithm enables the optimized power distribution, hence it is a more practical solution in networks with increased number of secondary users.
\end{abstract}

Keywords: Cognitive Radio Networks (CRN), Distributed Stochastic Algorithms (DSA), Distributed Algorithm for Power Control, Interference Constraints, Power Reduction Algorithm

\section{Introduction}

The enormous growth in the deployment of wireless communication, has led to the growth of various communication systems have come up in both licensed and unlicensed spectrum, suitable for different demands and various standards like the IEEE 802.11, Bluetooth, UWB, IEEE 802.16, etc. The non-line-of-sight applications uses the spectrum under $3 \mathrm{GHz}$. Hence by 2020 the advances in wireless communication requires radio spectrum allocation for many more devices included in the wireless systems ${ }^{1}$.

A high degree of utilization of the spectrum is shown in the allocation chart of the Federal Communications Commission (FCC). But, the FCC Spectrum Policy Task Force has reported the usage of the allocated spectrum in the non-line-of-sight radio propagation, ranging from $15 \%$ to $85 \%$ of the spectrum below $3 \mathrm{GHz}$ due to the vast temporal and geographic variations. The issues in spectrum utilization occur in the frequency range higher than the $3 \mathrm{GHz}$ range. A significant amount of spectrum is underutilized due to the mismanagement in the use of the assigned spectrum ${ }^{2}$.

The Cognitive Radio (CR) technology of dynamic spectrum access, where the licensed users share the wireless communication channel in an optimistic way. The mobile users are provided with higher bandwidth using heterogeneous wireless architectures through CRs and with the use of dynamic spectrum access techniques. The CR networks raise several challenges in the wide spectrum range and in the QoS requirements diversity for various applications. 


\section{Design Concepts and Requirements}

A CR network is considered as shown in figure 1, where the secondary users transmit the data in the presence of primary users. The interference between the primary and secondary users transmission can degrade the performance of the system. Both primary and secondary users share the same spectrum band for transmission.

The power minimizing algorithm can be applied to the CR system where considerable number of interference causing constraints are not satisfied and certain transmitting links are switched off. $\Psi$ represents the set with SINR constraint satisfied transmitting users and $\Phi$ represents the set with the users satisfying both SINR and interference constraints. There is no need for finding the set $\Phi$, if the interference constraints are not satisfied by the secondary users, as the two sets $\Phi$ and $\Psi$ are equal. When there is violation of certain interference constraints, then the elimination algorithm is employed to obtain $\Phi$ from $\Psi^{3}$.

The distributed algorithm for power control is used to calculate the values of $\Psi$. If the power minimizing algorithm cannot be implemented, certain number of secondary links are eliminated using the proposed distributed statistical elimination algorithm to satisfy the SINR requirement. The desired set of $\Phi$ is obtained as shown in Figure 2.

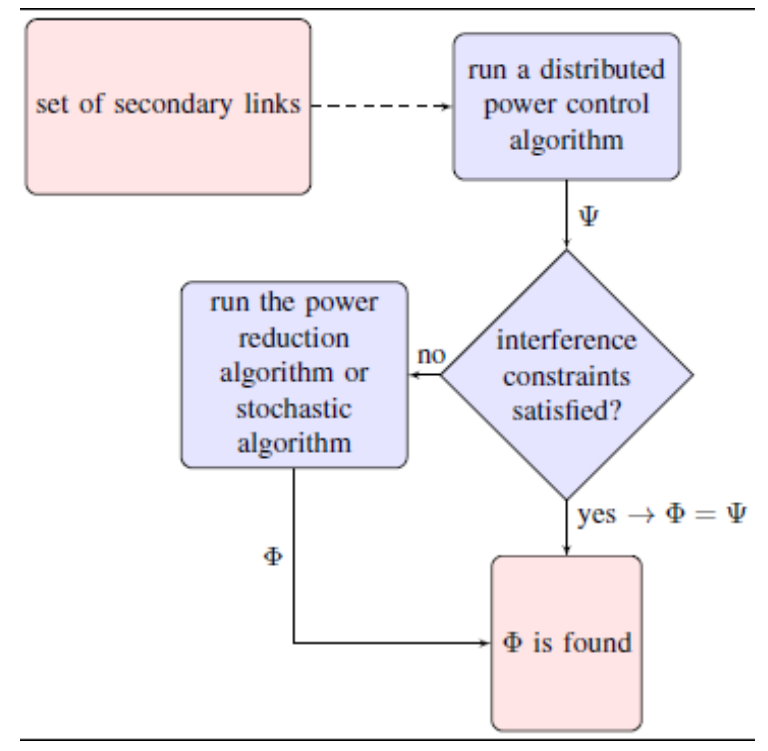

Figure 1. The proposed power control algorithm in a cognitive radio network.

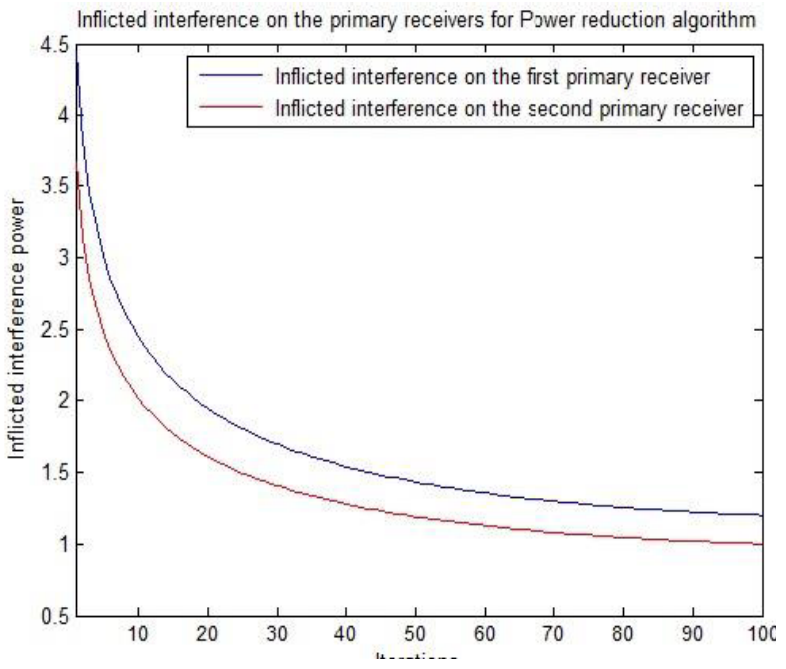

Figure 2. Power minimizing algorithm.

\section{Distributed Algorithm for Power Control}

Distributed algorithms for power control are derived from iterative algorithms. The following distributed power control scheme is obtained from the first-order Jacobian iterations,

$$
P_{i, s e c}(K+1)=\min \left\{p_{i}(k) \frac{\gamma_{i, s e c}^{\text {tar }}}{\gamma_{i, s e c}(k)}, p_{\text {sec }}^{\max }\right\}, i=1,2, \ldots . N
$$

Each node should only have knowledge on the self SINR received at the designated receiver so that the power transmitted is updated. The control channel provides the feedback from the receiving node, so that the algorithm is fully distributed. The algorithm should satisfy the convergence properties.

The Interference function $\mathrm{I}(\mathrm{p})$ is standardized when the following three conditions are satisfied: positivity, monotonicity and scalability. The convergence to a minimum equilibrium is achieved when the value of $\mathrm{p}(\mathrm{k}+\mathrm{l})=\mathrm{I}(\mathrm{p}(\mathrm{k}))$ corresponds to the minimum use of power. A special case of the iterative standard algorithm is the distributed algorithm for power control. The Jacobi iteration algorithm converges slowly as it is a iterative fixed-point method. As this method is simple, it is used for the equation selection in power control for CR networks. Many advanced algorithms with higher convergence speed are available in the literature 4 .

The inequality in the equation of the distributed 
algorithm for power control of CR networks will not force the primary users QoS requirements. Therefore if the equation is applied to the secondary users alone the QoS requirement will not be satisfied ${ }^{5}$.

\subsection{Power Control for Multiple Secondary users}

The solution to the power control using both centralized and distributed methods are provided in this section. The distances such as $d$ and $y_{i}$ are assumed to be estimated accurately with the help of geo-location devices (e.g. GPS), control signals, or spectrum sensing techniques in order to obtain the power and interference. These values are applied to calculate the power of the primary users with accurate estimation of the distances ${ }^{10-14}$.

\section{Proposed Power Minimizing Algorithm}

An proposed algorithm reduces the transmitted power by the set of $\Psi$ secondary users to decrease the interference constraints with the primary receivers as shown in figure 1. $\mathrm{P}_{\mathrm{i} 1}$ is the power transmitted by the $\mathrm{i}$-th secondary transmitter before applying the power minimizing algorithm and the $j$-th interference temperature is given by Qi. The value $g_{i j}$, denotes the gain of the channel power between the i-th secondary transmitter and the $j$-th primary receiver. If the set $\Psi$ has ' $r$ ' members and if 's' of the interference constraints which are violated by the members is given by,

$B_{i, \mathbf{1}}=\sum_{j=\mathbf{1}}^{r} p j 1$ gji $>Q i 1 \leq i \leq$

where $B_{i, 1}$ denotes the total interference of the $i^{\text {th }}$ primary receiver.

On the assumption, that the general algorithm for power control is applied to secondary users operating without the interference constraints. The general algorithm for power control reduces the power obtained by satisfying the interference constraints, but the secondary network SINR constraints should not be violated. The reduction in the power is done according to the following equation:

$p_{i \mathbf{2}}=p_{i \mathbf{1}}-\delta a_{i} \quad i=1,2, \ldots \ldots n$ $\delta$ is the constant and $\alpha_{i}$ are the coefficients of the algorithm.

$\mathrm{h}_{\mathrm{ij}}$ is the channel power gain between the $\mathrm{i}^{\text {th }}$ secondary transmitter and the $j^{\text {th }}$ secondary receiver and $\eta_{i}$ is the noise power at the $\mathrm{i}^{\text {th }}$ channel. If ' $\mathrm{k}$ ' is the total interference between the primary transmitters and the secondary receivers, then the SINR before applying the power minimizing algorithm is

$\gamma_{i \mathbf{1}}=\frac{\boldsymbol{h}_{i i} p_{i 1}}{\sum_{j=1, j \neq i}^{n} \boldsymbol{h}_{i j} p_{j \mathbf{1}}+\eta i+k}, i=1,2 \ldots . n$

After applying the power minimizing algorithm the gain is given by,

$\gamma_{i \mathbf{2}}=\frac{\boldsymbol{h}_{i i} p_{i 2}}{\sum_{j=1, j \neq i}^{n} \boldsymbol{h}_{i j} p_{j \mathbf{2}}+\prod i+k}, i=1,2 \ldots n$

Assuming that the general algorithm for power control is implemented on the secondary users and if the SINR threshold level is represented by $\gamma_{\text {th }}$ then the SINR level of the members of the set $\Psi$ should be greater than $\gamma_{\mathrm{th}}$. As a result

$\gamma_{i 1} \geq \gamma_{t h}, i=1,2, \ldots, n$

The proposed power minimizing algorithm should increase the SINR by maintaining the interference constraints. Therefore, the value of $\gamma_{\mathrm{i} 2}$ has to be greater than $\gamma_{\mathrm{i} 1}$ and since according to (6), $\gamma_{\mathrm{i} 1}$ itself is greater than $\gamma_{\text {th }}$, always the value of $\gamma_{\mathrm{i} 2} \geq \gamma_{\mathrm{th}}$, where the interference constraints are still maintained. The value of power obtained from (3) is replaced, the requirement $\gamma_{\mathrm{i} 2} \geq \gamma_{\mathrm{i} 1}$, can be simplified to

$\gamma_{i \mathbf{1}}=\frac{\boldsymbol{h}_{i i} a_{i}}{\sum_{j=1 . j \neq \mathbf{1}}^{n} \boldsymbol{h}_{i j} a_{j}}, i=1,2 \ldots . n$

Hence, using the power minimizing algorithm the coefficients of $\alpha_{i}$ should be found to satisfy (7). Since $\gamma_{i 1}$ is assumed to be greater than $\gamma_{\mathrm{th}}$, its value is further set to satisfy the condition in such a way that

$\gamma_{\text {th }} \geq \frac{\boldsymbol{h}_{i i} a_{i}}{\sum_{j=1 . j \neq \mathbf{1}}^{n} \boldsymbol{h}_{i j} a_{j}}, i=1,2 \ldots . n$

Then (8) will be derived to maintain the SINR constraints. The above mentioned requirements is represented using the vector space matrix T, given by

$T_{i j}= \begin{cases}-\boldsymbol{h}_{i i}, & \text { if } i=j \\ \gamma_{t \boldsymbol{h}} \boldsymbol{h}_{i j} & \text { if } i \neq j\end{cases}$ 
$A=\left[a_{1}, a_{2}, \ldots a_{n}\right]^{T}$

$T A \geq 0$

$A \geq 0$

Therefore, in order not to violate the SINR constraints using the power minimizing algorithm at each stage the SINR constraints are calculated, to find a vector satisfying the equations (10) and (11). Though the representation is not complex, a regular linear algebraic algorithm can be the search algorithm but a simpler solution is proposed for large practically important networks. If the coefficients are selected such that,

$a_{i}=\frac{\sum_{j=1 . j \neq 1} \boldsymbol{h}_{i j}}{\boldsymbol{h}_{i i}}, \quad \quad i=1,2 \ldots . . n$,

$=\frac{1}{n-1}\left(\frac{h_{\max }}{h_{\min }}\right)^{2}$

Where $h_{\max }$ and $h_{\min }$ are the maximum and the minimum channel power gains, respectively. When the users of the network are more than the threshold level. The proposed power minimizing algorithm is modified as follows:

- The members of set $\Psi$ transmit with powers $\mathrm{P} 11, \mathrm{P} 21, \ldots . . \mathrm{Pn} 1$ from the general algorithm for power control.

- The algorithm reaches the end if interference constraints are not violated. Otherwise, the gain coefficients must be chosen as,

$$
a_{i}=\frac{\sum_{j=1 . j \neq \mathbf{1}} \boldsymbol{h}_{i j}}{\boldsymbol{h}_{i i}}, \quad i=1,2 \ldots . . n
$$

- The $\delta$ value is chosen to satisfy (13) with consideration on the lower bound of detection

- The vector of the power is updated as

$p_{i \mathbf{2}}=p_{i \mathbf{1}}-\delta_{a i}, \quad i=1,2, \ldots, n$

- The interference constraints are checked and if there is any violation go back to step (2). If there is no violation of the interference constraints the algorithm stops.

\subsection{The Control of power for a Single Secondary Transmitter}

The study on a single secondary user considered for simplicity. The problem is validated to judge the feasibility of applying the power control algorithm. The analysis is done under the assumption that the power received is a function of the transmitted power and path loss without taking into account the fading effects such as shadowing and small-scale fading. Let $a_{1}$ and $a_{2}$ represent the path loss of the Television transmitter and cognitive radio transmitter respectively. Due to the several hundred meters difference in the antenna heights of the two transmitters the path loss of the Television transmitter $\left(a_{1}\right)$ is smaller than path loss of the cognitive radio transmitter $\left(a_{2}\right)$. The modulation schemes and waveform design, the orthogonality factors etc., are the factors influencing the interference between the primary and secondary users ${ }^{6}$.

Based on these assumptions, the SINR of the television receiver at the worst location of the television coverage area and the interference of the secondary receiver of the cognitive radio transmitters are compared $d^{7-9}$.

\subsection{Distributed Statistical Elimination Algorithm}

To analyze the algorithm, the transmitters should set the values for power individually based on the local information along with the general information from the network. There is no need for extra information from other transmitters. The values of quantities such as $P_{i}, Q_{j}$ $, B_{j}, g_{j i}$ for $j=1,2, \ldots . m$ should be given to the $i^{t \text { th }}$ transmitter. The values of the $j^{\text {th }}$ primary receiver total inflicted interference and the channel gains are obtained from the sensors located close to the primary user from where the information is fed back to secondary transmitters.

The proposed algorithm to obtain the set $\Phi$ from $\Psi$ is as follows:

- The transmission of all the members of set $\Psi$ start the transmission with powers $\mathrm{P}_{1}, \mathrm{P}_{2}, \ldots \ldots \mathrm{P}_{\mathrm{r}}$.

- The feedback of the total interference near the primary receivers is transmitted to the secondary users.

- Set $\Phi=\Psi$, if the interference constraints are not violated then the algorithm comes to a stop. If there is any violation it goes into the next step.

- Through the elimination algorithm the information of $\Phi$ from $\Psi$ is extracted.

- In the following time slots when other links are not transmitting, the measurement of the primary receivers status of interference is feedback to the secondary transmitting links. 
- The algorithm stops when the all the interference constraints are satisfied in this time slot. Otherwise, the transmission should be stopped on the other links to ensure that the interference levels are within the constrained level so the algorithm goes back to step (4).

The distributed statistical elimination algorithm is described as follows:

If the interference constraints are not satisfied, the probability of the $\mathrm{i}^{\text {th }}$ secondary transmitter is found as follows:

The transmission of the $\mathrm{i}^{\text {th }}$ secondary transmitter stops with probability of $\mathrm{W}_{\mathrm{i}}$. The probability $\mathrm{W}_{\mathrm{i}}$, depends inversely on the total power of interference in the primary receivers. It is directly proportional to the transmitted power of the $\mathrm{i}^{\text {th }}$ secondary transmitter. This value is high for the secondary users with more interference at the primary receivers. Thus the links with higher probability should be removed.

But care should be taken before the removal of these links. There is a possibility of many secondary users to stop transmitting without being actually stopped by the algorithm at a particular time. Thus there is an ambiguity in obtaining the from the set $\Psi$ set of best values for $\Phi$.

A unique set of values cannot be derived as every individual run gives different set of values for $\Phi$ due to the statistical property of the algorithm. Hence the number of iterations of the algorithm should be increased to find the set of best values for $\Phi$ maximizing the selected number of links.

There is also another concern on the increase in the SINR level greater than the threshold level. This occurs as the members of set $\Phi$ stop transmitting before the algorithm stops the SINR level on some of the links which are still transmitting increases thereby resulting in greater SINR values.

\section{Simulated Results and Discussion}

Simulation is done using MATLAB to compare the performance of different algorithms.

The power control algorithm is applied to the secondary users network, where the interference level of the users in set $\Psi$ is greater than the threshold interference level given by $\gamma_{\text {th }}$ as a result as shown in Figure 3 .
When the set $\Psi$ has five users transmitting with the power levels given as below:

$\llbracket\left[p \rrbracket_{11}, p_{21}, p_{31}, p_{41}, p_{51}\right]=1,1.3,0.5,2,1.3$

At the same time five secondary users trying to transmit the data to five secondary receivers.

When there are only two primary users with interference temperatures $[\mathrm{Q} 1, \mathrm{Q} 2]=[3,2.5]$ the interference constraints are not satisfied. The power gains of the channels for the two primary users is as follows

$$
\begin{aligned}
& \llbracket\left[h \rrbracket_{11}, h_{21}, h_{31}, h_{41}, h_{51}\right]=[0.9,0.8,0.7,0.6,0.2] \\
& \llbracket\left[h \rrbracket_{12}, h_{22}, h_{32}, h_{42}, h_{52}\right]=[0.5,0.8,0.1,0.2,0.1] \\
& \llbracket\left[h \rrbracket_{13}, h_{23}, h_{33}, h_{43}, h_{53}\right]=[0.3,0.8,0.9,0.4,0.2] \\
& \llbracket\left[h \rrbracket_{14}, h_{24}, h_{34}, h_{44}^{\prime} h_{54}\right]=[0.5,0.4,0.6,0.7,0.3] \\
& {\left[h_{15}, h_{25}, h_{35}, h_{45}, h_{55}\right]=[0.1,0.3,0.4,0.9,0.5]}
\end{aligned}
$$

If there are only two primary users with interference temperatures $[\mathrm{Q} 1, \mathrm{Q} 2]=[3,2.5]$ whose interference constraints are not satisfied. If the channel power gains for these two primary users are given as below

\section{$\llbracket\left[g \rrbracket_{11,}, g_{21}, g_{31}, g_{41}, g_{(51]}\right)=[0.7,0.8,0.4,0.9,0.2]$}

The total interference between the secondary transmitters and the primary receivers before applying the proposed algorithm is given by $[\mathrm{B} 11, \mathrm{~B} 21]=[4,3.76]$.

After executing the distributed statistical elimination algorithm for the first time, the power vector is given by

$\llbracket\left[p \rrbracket_{12,} p_{22,}, p_{32,} p_{42,} p_{52,}\right]=[0.1 .3,0.5,2,0]$

When the transmission of the first and the last transmitters stops, the total interference levels after running the distributed statistical elimination algorithm is given by $[\mathrm{B} 12, \mathrm{~B} 22]=[1.94,1.32]$. It is observed that these values are very much lower than the given interference temperatures $[\mathrm{Q} 1, \mathrm{Q} 2]=[3,2.5]$.

When the algorithm is executed more than once, the power vector with the maximum occurrences is selected. The algorithm is then run a number of times, by selecting the best value for the power vector each time, the obtained levels of interference are plotted in Figure 4. 
In figure 3 , the probability of convergence is plotted as it increases with the increase in the number of iterations.

The power minimizing algorithm is executed for the parameter $=0.0083$ maintaining the same values for the power and the channel gains. Figure 3 shows the relation between the obtained power of interference on the primary receivers to the number of iterations. The statistical nature of the algorithm decreases from the previous algorithm as it is reduces linearly with the increasing interference levels. The reduction in interference power leads to a constant value for the power vector. The comparison of interference between the first primary receiver and second primary users of various algorithms is shown in figure 4 and figure 5.

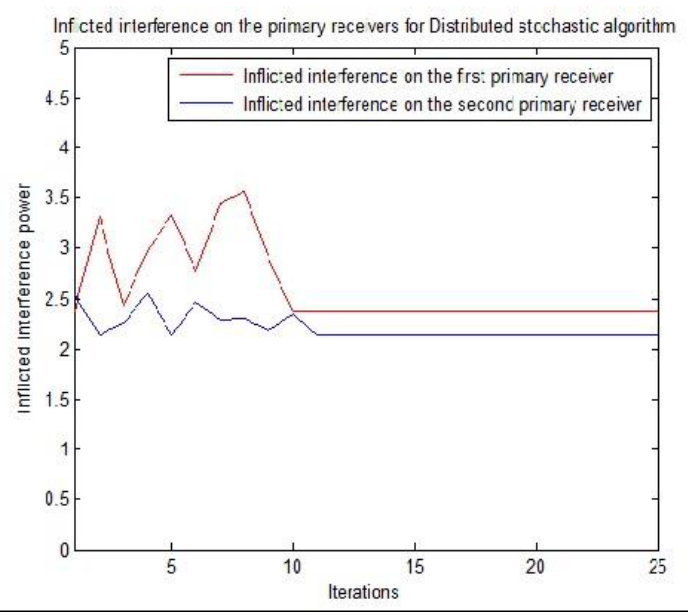

Figure 3. Distributed stochastic elimination algorithm.

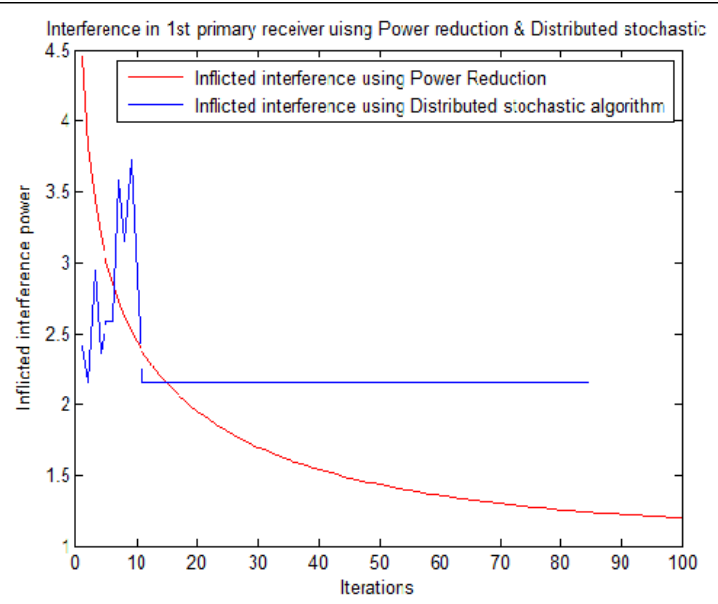

Figure 4. Difference of power reduction and distributed stochastic elimination algorithm.

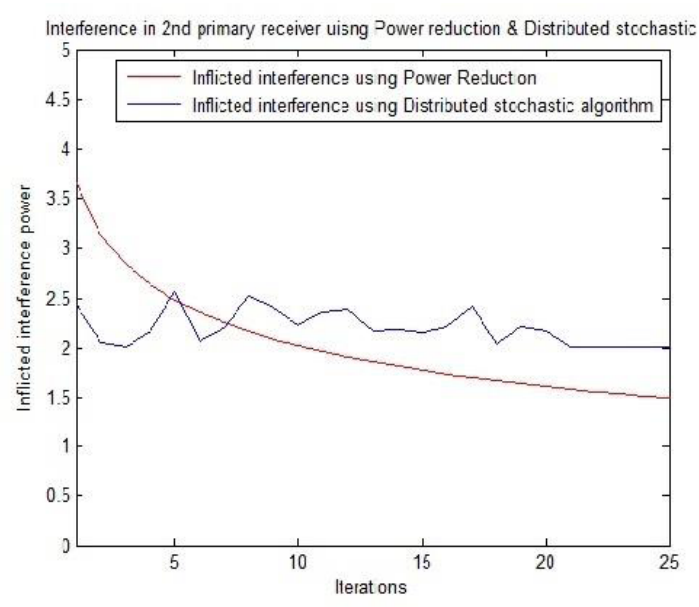

Figure 5. Difference of power reduction and distributed stochastic elimination algorithm.

\section{Conclusion and Future Work}

The distributed algorithm for power control does not force the equality of the QoS requirements of primary users. Thus the QoS requirement of the primary users may be violated if the algorithm is applied to the secondary users alone. The resultant SINR level for all the links of a wireless network is not constant as the power of transmission related to one link may increase the level of interference on other links. The transmitting links causing the increase in interference must be turned off to maintain the threshold SINR levels of the remaining links. The power minimizing algorithm is introduced to reduce the interference temperature. A good solution for the SINR values should be achieved in such a way that it does not exceed the threshold SINR value using the distributed algorithm for power control. A distributed statistical algorithm for power control incorporated to reduce the number of secondary users in order to satisfy the SINR requirement for all the other links.

\section{References}

1. Mitola J. Cognitive radio for flexible mobile multimedia communication, in Proceedings of IEEE International Workshop on Mobile Multimedia Communication (MoMuC), San Diego, CA, USA, 1999; 3-10.

2. Kolodzy PJ. Spectrum policy task force report. Federal Communications Commission. 2002. 
3. Foschini GJ, Miljanic Z. A Simple Distributed Autonomous Power Control Algorithm and its Convergence. IEEE Transactions on Vehicular Technology. 1993 Nov; 42(4):641-46.

4. Grandhi SA, Zander J, Yates R. Constrained Power Control Wireless Personal Communication. 1995; 1(4):257-70.

5. Koskie S, Gajic Z. A Nash Game Algorithm for SIR-Based Power Control in 3G Wireless CDMA Networks. IEEE/ ACM Transactions on Networking. 2005 Oct; 13(5):101726.

6. Holliday T, Bambos N, Glynn P, Goldsmith A. Distributed Power Control for Time Varying Wireless Networks: Optimality and Convergence in Proceedings of Allerton Conference. 2003. p. 1-10.

7. Andersin M, Rosberg Z, Zander J. Gradual Removals in Cellular PCS with Constrained Power Control and Noise. Wireless Networks. 1996 Mar; 2(1):27-43.

8. Rasti M, Sharafat AR, Zander. Improved Distributed algorithm for power controls with Gradual Removal in Wireless Networks. 14th European Wireless Conference, (EW2008), Prague, 2008 Jun 22-25. p. 1-5.

9. Rasti M, Sharafat AR, Zander J. A Distributed and Efficient Power Control Algorithm for Wireless Networks. IEEE In- ternational Symposium on Personal, Indoor and Mobile Radio Communications, PIMRC'08, Cannes, 2008; 1-6.

10. Qian L, Li X, Attia J, Gajic Z. Power Control for Cognitive Radio Ad Hoc Networks. Proceedings of the 15th IEEE Workshop on Local and Metropolitan Area Networks, LANMAN'07, Princeton, NJ. 2007 Jun 10-13; 7-12.

11. Kumar BS, Srivatsa SK. An Efficient Spectrum Sensing Framework and Attack Detection in Cognitive Radio Networks using Hybrid ANFIS. Indian Journal of Science and Technology. 2015 Oct; 8(28):1-12.

12. Padmavathi G, Shanmugavel S. Performance Analysis of Cooperative Spectrum Sensing Technique for Low SNR Regime over Fading Channels for Cognitive Radio Networks. Indian Journal of Science and Technology. 2015 Jul; $8(16): 1-5$.

13. Rajesh PN, Muthaiah R. Carrier Synchronization in Software Defined Radio using Costas Loop. Indian Journal of Science and Technology. 2013 Jun; 6(6):4697-701.

14. Nanthini SB, Hemalatha M, Manivannan D, Devasena L. Attacks in Cognitive Radio Networks (CRN) - a Survey. Indian Journal of Science and Technology. 2014 Apr; 7(4):530-36. 